\title{
Research on Thermal Aging Properties of Composite Insulating Materials in High Voltage Switchgear
}

\author{
Yongyong $\mathrm{JIA}^{1, \mathrm{a}^{*} \text {, Yanjun JIANG }}{ }^{2, \mathrm{~b}}$, Jinggang $\mathrm{YANG}^{1, \mathrm{c}}$, Guogang ZHANG ${ }^{2, \mathrm{~d}}$ \\ ${ }^{1}$ State Grid Jiangsu Electric Power Company Research Institute, Nanjing 211103, China, \\ ${ }^{2}$ State Key Lab of Electrical Insulation and Power Equipment Xi'an Jiaotong University, Xi'an \\ 710049, China \\ a15105168898@163.com, balicejiang226@163.com, c15105168828@163.com, \\ ggzhang@mail.xjtu.edu.cn
}

\begin{abstract}
Keywords: high voltage switchgear; composite insulating materials; thermo-oxidative aging; dielectric properties.
\end{abstract}

Abstract: Electrical properties, thermal stability and flammability are the important properties of insulating material in the high voltage metal-enclosed switchgear, which can affect the insulation fault of switchgear. With the development of new composite materials, it has a revolutionary impact on the switchgear insulation. In this paper, the four composite insulating materials, which are BMC, SMC, GPO-3 and epoxy resin composite, have been aged under different periods up to 1200 hours. The dielectric properties and physical and chemical property were investigated at different aging stages. The performances of the four materials and the reason of its degradation have been analyzed. According to the experiment results, with the increase of aging time, their dielectric properties are always decrease firstly, increase secondly and keep stable finally. The dielectric properties of epoxy composite are worst. Thermo-oxidative aging usually leads to the weak bond of polymer reacting with oxygen. Epoxy composite has the highest melting temperature. GPO-3 has the best flammability. BMC is well-crystallized, which has the best anti-aging properties. This study provides a basis to choose the suitable dielectric materials for the design of high voltage switchgear.

\section{Introduction}

The requirement of power quality is higher and higher in modern power systems, it means the reliability and stability of the power equipment must have the higher quality. Switchgear is one of the most important power equipment, stable operation of which affects the continuous and reliable supply of power. CIGRE has done the switchgear failure statistics, the results show that $40 \%$ of the reason is caused by inner insulation defects of switchgear. At the same time, some similar statistics have been done in China, which show that the insulation faults account for $37.3 \%$ in the entire switchgear failure ${ }^{[1,2]}$. In recent decades, with the change of switchgear internal structure, In recent decades, with the constant change of switchgear internal structure, its internal space is gradually reduced, and the load current is also rising. The primary reason of switchgear accident is due to the severe fever inside the equipment. Insulation material is always working in the environment with high temperature, damp and filthy, so the insulating properties of the material are constantly aging. It makes insulating property degradation, leads to corona discharge and flashover, which may threaten the stability and safety of the device ${ }^{[3,4]}$. Therefore, studying the aging properties of insulating materials has important significance, such as to ensure the stable operation of equipment, to reduce the number of maintenance, to improve the reliability of power system.

With the advent of the glass fiber, the insulating and anti-aging properties of the composite material are improved greatly using the intensifier of composite. It has brought the revolutionary change in the insulation of high voltage switchgear. The equipment working at different situations has different demands to the insulating properties of composite materials. Therefore, studying a variety of composite insulation material properties after aging and balancing the good points against the bad points of their dielectric properties, physical and chemical properties have an important significance for the optimization design of the future switchgear. The material in switchgear mainly affected by the heat of cabinet and load current. Under the participation of the oxygen in the air, the 
main reason of material degradation is thermos-oxidative aging. This paper studies on four kinds of composite insulation materials, which are epoxy composite, BMC, SMC and GPO-3. It investigated their dielectric properties, physical and chemical properties at different artificial thermo-oxidation aging stages. According to the experiment results, it discusses the performance degradation rule of different kinds of composite insulation materials under thermos-oxidative aging, and analyzes the causes of material performance degradation. It compares the advantages and disadvantages of them in order to providing a strong basis for the optimization design of the future switchgear.

\section{Experimental}

\section{(1)Materials Selection}

BMC (bulk molding compound) is manufactured by mixing UPR (unsaturated polyester resins), chopped glass fiber and other packing and additives, which has excellent electrical properties and mechanical properties, high temperature resistance and corrosion resistance. BMC has simple molding process. By adjusting the ratio of glass fiber length and resin system, it can be processed into different products to adapt the corresponding requirements ${ }^{[5]}$.

SMC (sheet molding compound) is a ready to mold glass fiber reinforced polyester material primarily used in compression molding and mixed with LPA and some other packing. SMC also has good electrical performance. But SMC is superior in water absorption and impact resistance.

GPO-3(polyester glass mat) consists of alkali-free glass mat impregnated and bonded with UPR, and laminated under high temperature and high pressure. It can keep good electrical performance and mechanical performance under the condition of high temperature and humidity. What's more, the performances of arc resistance and tracking and erosion resistance are excellent, and it is widely used in the breakers and motors ${ }^{[6]}$.

Epoxy composite consists of glass-fiber fabric and epoxy resin. Epoxy resin contains polar hydroxy, ether bond and epoxy group. Under proper conditions, it can form three-dimensional crosslinked reticular compounds by adding curing agent. Its chemical construction decides that epoxy resin, curing agent and modified compound can adapt to any requirements, such as low shrinkage, high adhesion strength, high dielectric strength and good mechanical properties ${ }^{[7,8]}$.

\section{(2)Scheme}

Artificial aging experiment is a short time period and efficient method, which can be used to simulate the materials long-term working in the actual case. By accelerating artificially the rate of aging of insulation materials, it can explore the aging characteristics of the material in a short time. In this experiment, the four kinds of composite materials have been aged, as mentioned above, which are epoxy composite, BMC, SMC and GPO-3. Because the specific standard on choosing the aging temperature of the composites has not appeared yet, the experiment will set the aging temperature under the general principle. The principle is that the aging temperature of resin matrix composite materials must be lower than the glass transition temperature of resin.

tABle I. THE EXPERIMENTAL SCHEME Of ARTIFICIAL THERMO-OXIDATION AGING

\begin{tabular}{|c|c|c|}
\hline Aging Period & Aging Time $(\mathbf{H})$ & Aging Temperature $\left({ }^{\circ} \mathrm{C}\right)$ \\
\hline 1 & 120 & 70 \\
\hline 2 & 120 & 90 \\
\hline 3 & 120 & 110 \\
\hline 4 & 120 & 110 \\
\hline 5 & 240 & 110 \\
\hline 6 & 240 & 110 \\
\hline 7 & 240 & 110 \\
\hline
\end{tabular}

The test-pieces of composite material were put into aging oven and aged by several cycles. As shown in Table.1, the first two cycles are the transition period in early aging. From the third cycle, it enters the high-temperature aging stage. The aging time is totally $1200 \mathrm{~h}$. Each end of an aging period, the test-pieces will be taken out from the aging oven to test the dielectric, physical and chemical properties, and then put back to be aged continued. According to the rough estimation, 
from the beginning of the normal operating temperature, if material temperature is increased by $10^{\circ} \mathrm{C}$, its lifetime will be cut in half. After the entire aging test, the test-pieces of four materials are equivalent to serve about 20 years under the normal condition.

\section{(3)Measurement}

- Dielectric properties test of insulating material

The electrical property is the core of the electrical insulating properties, which must be considered first. All other properties must meet the electrical requirements as the premise. The experiment is to measure two primary electrical parameters of the materials, which are the relative dielectric constant and tangent of dielectric lose angle. It can observe the electric insulating intensity, the moisture content and the aging depth from the macroscopic level. The equipment of type 2801 Schering Bridge was selected, which can measure the dielectric properties of material parameters under the power frequency. Its measurement range of capacitance $C_{x}$ is $0.01 \mathrm{pF} \sim 0.1 \mathrm{pF}$, its measurement range of the tangent of dielectric lose angle tan $\delta$ is $1.0 \times 10^{-6} \sim 3.5$.

- The physical and chemical properties test

According to the physical and chemical properties of materials, the process of thermos-oxidative aging is always accompanied by some endothermic and exothermic reactions. It can reflect the aging rates of materials by measuring the reaction heat flow under certain temperature. And then research the aging state of insulation materials from the perspective of the aging mechanism. In this experiment, using AG135 photoelectric balance to weigh 10mg samples approximately, the melting and decomposition of materials were analyzed by 822e DSC. The temperature range of test is $25^{\circ} \mathrm{C}$ $\sim 400^{\circ} \mathrm{C}$. The rate of temperature rising is $20^{\circ} \mathrm{C} / \mathrm{s}$. According to thermal enthalpy difference between the reference sample and the measurement sample, it can be used to measure the heat flow curve along with the change of temperature.

\section{Results And Discussion}

\section{(1)Dielectric Property}

As before, each end of an aging period, the materials will be taken out from the aging oven and tested the performance, and then put them back to age continued. The test results are shown as follow. Figure 1 shows that the relative permittivity of four materials (epoxy resin composite, BMC, SMC, GPO-3) change along with the aging time. Figure 2 shows that the dielectric loss tangent of four materials change along with the aging time. In the figures, the first data of each curve is the dielectric parameters of original materials, which are without aging. The rest data are the test values in the corresponding aging cycles.

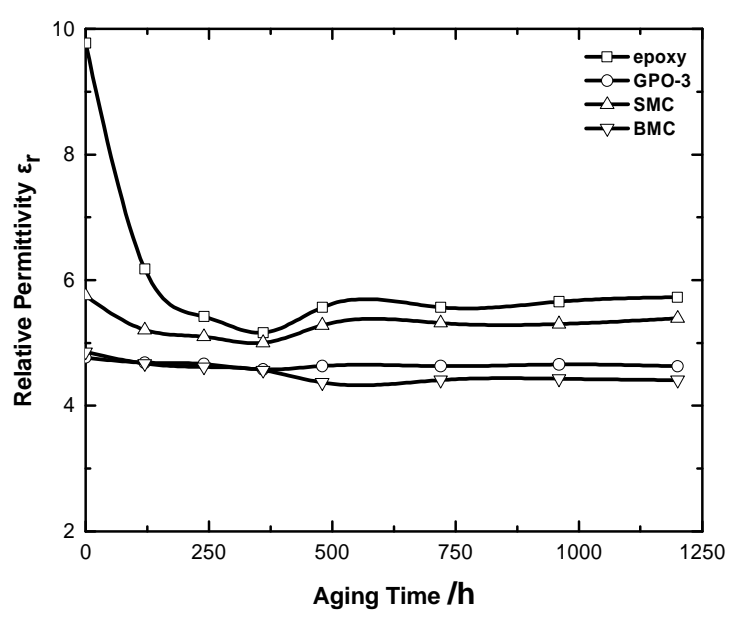

Fig. 1. Relative permittivity of four materials versus aging times

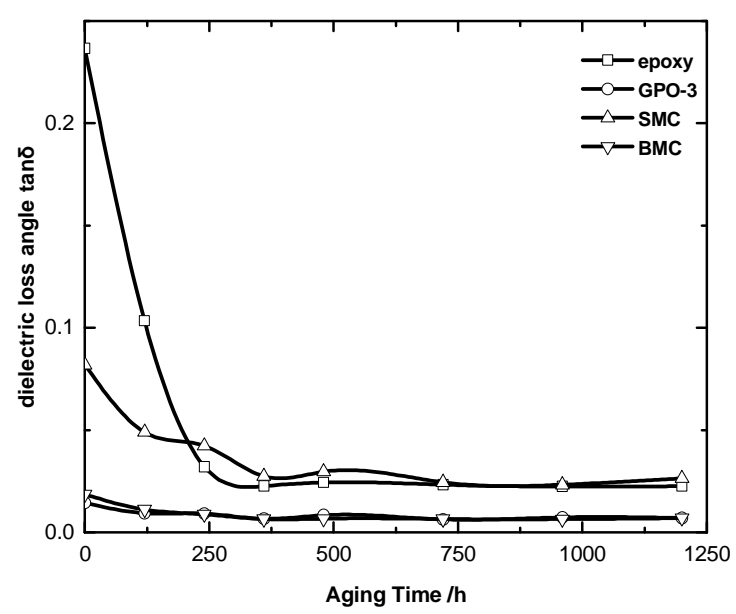

Fig. 2. Dielectric loss angle tanget of four materials versus aging times 
As it shows in figure 1, in the early part of the aging, with the increase of aging time, the values of relative permittivity and dielectric loss tangent of the four materials are decreased continuously. In addition, the values of dielectric parameters decrease rapidly in the first two periods, and then they change gently. This is mainly because that, the test-pieces of composite materials always have some certain initial moisture content when placed at room temperature after a period of time. As a consequence, the volatilization of material initial moisture content results in the rapid decline of dielectric parameters.

The thermo-oxidative aging process of thermosetting resin matrix composite contains dehumidification, solidification and disintegration. In other words, the insulation materials are in physical aging and chemical aging at the same time. The role of physical aging is to make the material matrix free volume shrinkage, which leads to the increase of density. The polarization of dielectric becomes more difficult, and then the relative permittivity and dielectric loss tangent will decrease. In the chemical aging of polymer, weak bond of organic molecules can easily react with oxygen. The reaction causes the degradation and crosslinking of macromolecular chains. It produces large amounts of hydrogen peroxide with polar group, and these lead to the increase of dielectric parameters.

The changed trend of experimental results in figure 2 shows that the physical aging effects are greater than the chemical aging effects in the first three aging periods. The dielectric polarization increases difficulty. The dielectric parameters of insulation materials are on the downward trend in general. From the beginning of the fourth aging cycles, the dielectric parameters show an increasing trend. The rising values mean that, the chemical aging effects are obvious, and high temperature increases the relaxation loss of polar groups at the stage of high temperature aging. As the aging time goes on, the coupling of free radical is terminated. Thermo-oxidative aging gradually into the final steady state, the corresponding dielectric parameters also remained unchanged.

Comparing the measurements of the four materials, it can also find that, the relative permittivity and dielectric loss tangent of epoxy composite drop greatly, which are the most sensitive to thermooxidative aging. And SMC is next. The performance of BMC and GPO-3 are stable relatively, and both of them have lower dielectric loss, which are relatively ideal insulation material of switchgear. GPO-3 has the smallest change of dielectric properties in these four materials. This is because that GPO-3 contains alkali-free glass fibers. For the glass fiber, alkali is with less, the electrical insulation properties and chemical stability of its polymer is better.

\section{(2)Physical and Chemical Characteristics}

Generally speaking, DSC (differential scanning calorimetry) can perfectly transform the temperature of the high polymer in three forms, which are the viscous flow state, high elastic state and glassy state. But for composite materials, due to theirs numerous components, it is difficult to completely measure the composition of composite materials. Even so, measuring the thermal conversion process of different composite materials can help to understand the physical and chemical properties of materials. From figure 3 to figure 6, the DSC curves of the four kinds of composite material are given, which are the changing rule of different thermal aging time. Trough of the curve stands for the melting process of material. And its area stands for the heat absorbed in the total process. Its peak value stands for the melting temperature. 


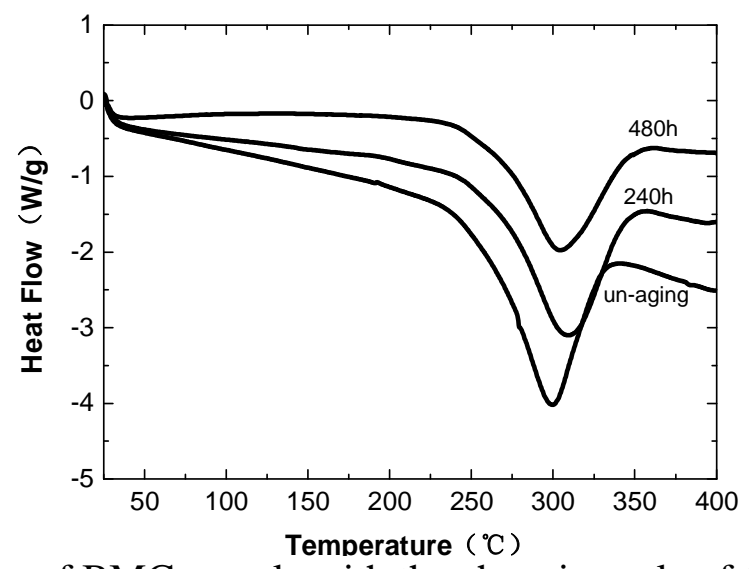

Fig. 3. The curve of BMC sample with the changing rule of the aging time

It can be seen from figure 3, for the BMC sample without aging, trough appears around $300^{\circ} \mathrm{C}$, which means BMC sample produces endothermic reaction. In other words, if BMC is working at the temperature reached about $300^{\circ} \mathrm{C}$, the matrix inner materials will happen thermal decomposition reaction in order to prevent the material temperature rising further by absorbing heat. And then it prevents material burning due to work in the high temperature for a long time. Therefore, the area of trough stands for the ability of flame-resistant to a certain extent. Comparing the three DSC curves at different aging time, it can be found out that, the aging time is longer, the melting temperature of BMC is higher, but area of the melting peak is smaller. It indicates that, due to the reactions with oxygen and heat accumulation effect, it causes the degradation and crosslinking of macromolecular chains during the process of aging. Narrowing of the melting peak area means that its absorption of heat is in reducing. Namely, within the material, the ability of preventing temperature rising further is decreasing, which manifests that its flame retardant ability is continually lower along with the aging.

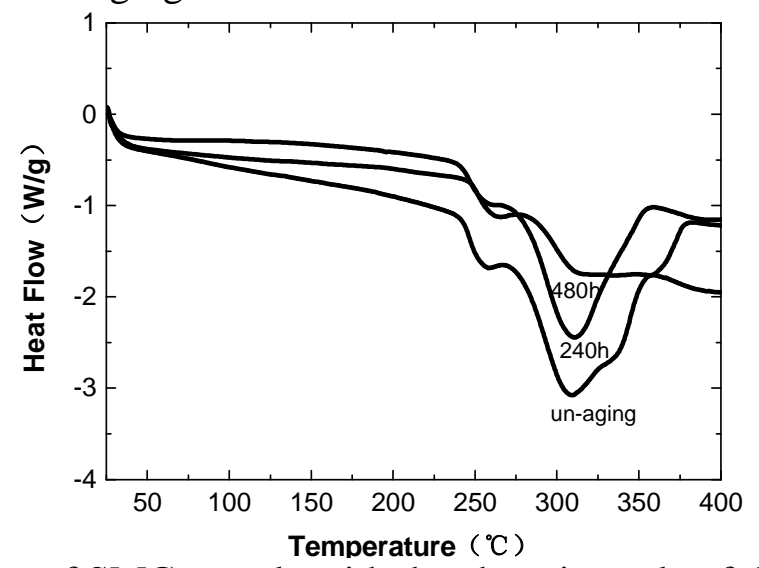

Fig. 4. The curve of SMC sample with the changing rule of the aging time

Figure 4 is the DSC curve of SMC sample. Comparing with Figure 3, what SMC is different from $\mathrm{BMC}$ is that, the curve of SMC appears many irregular troughs at temperature range of $250^{\circ} \mathrm{C}$ $\sim 350^{\circ} \mathrm{C}$. This is because that SMC contains both UPR and amount of LSA (like polystyrene) in order to reduce the crack in the compression molding. When $\mathrm{SMC}$ works at $250^{\circ} \mathrm{C} \sim 350^{\circ} \mathrm{C}$, its various components will decompose in different extent. Comparing with BMC, SMC can absorb more heat, but the decomposition temperature of SMC is lower than BMC. Therefore, thermal stability of BMC is better.

At the same time, comparing the three DSC curves of SMC at different aging degree, it can be found that, with the increase of aging time, the melting temperature of the SMC is moving to the direction of high temperature, but the area of melting peak becomes smaller. The DSC curve of SMC sample, which has been aged for 480 hours, is characterized by step-shaped after $300^{\circ} \mathrm{C}$. That means its specific heat capacity is changing. It indicates that the thermo-oxidative aging damages the crystallization of insulating materials. 
Overall, adding plenty of LSA, SMC has successfully reduced the cracks in the molding process and improved the production technology of materials. But it is found that LSA can produce a variety of different decomposition reaction with oxygen in the thermal analysis, and the temperature of first decomposition reaction is lower. So the thermal stability of SMC is inferior to BMC to some extent.

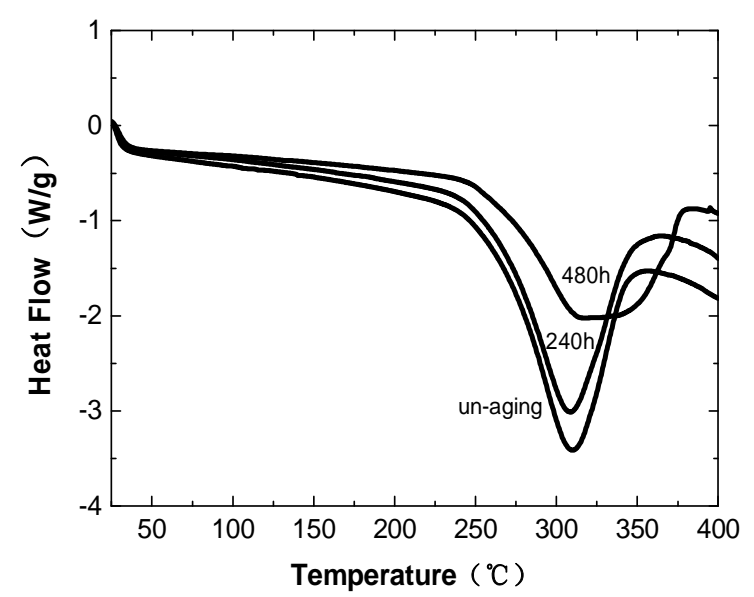

Fig. 5. The curve of GPO-3 sample with the changing rule of the aging time

Figure 5 is the DSC curve of GPO-3 sample. Comparing the test results of the BMC and GPO-3, it is easy to found out that the changing trend of experimental measurement curves is very similar. This is because the main material of BMC and GPO-3 are glass fiber and UPR. The trough appeared around $310^{\circ} \mathrm{C}$. Along with the increase of aging time, the melting temperature of the GPO-3 is also moving to the direction of high temperature, and the area of melting peak becomes smaller as well. The melting temperature of GPO-3 is higher than BMC. But while GPO-3 aged for 480 hours, the DSC curve of GPO-3 is characterized by step-shaped on the wave trough. It indicates that the thermo-oxidative aging damages the crystallization of insulating materials. Comparing with BMC, the area of GPO-3's melting peak is larger. It shows that GPO-3 absorbs more heat than BMC when melting. Although thermal stability of GPO-3 is not as good as BMC, the flame retardant is better.

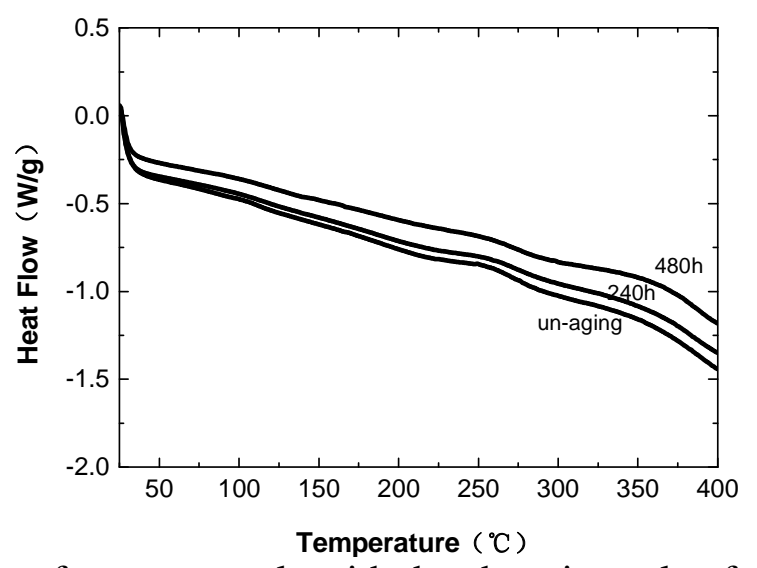

Fig. 6. The curve of epoxy sample with the changing rule of the aging time

Figure 6 is the DSC curve of epoxy composite sample. It shows a huge difference from the former, which is the heat flow is declining all the time, and the melting peak has occurred yet until $400^{\circ} \mathrm{C}$, the upper limit temperature of testing. Because the chemical structure of various types of epoxy resin, curing agent, and their modified compounds determine that they can almost adapt to any requirement from the low viscosity to high melting point. The molecular weight and the glass transition temperature of the epoxy resin samples are high, which help to enhance the thermal stability of materials. 


\section{Conclusion}

This paper made a study of artificial accelerated thermo-oxidative aging experiment on the four kinds of composite materials (BMC、SMC、GPO-3 and epoxy composite). the dielectric properties and the physical and chemical properties of these materials have been measured and analyzed. Some conclusions can be obtained as following.

(1) These four kinds of composite materials have the reaction of dehumidification, solidification and disintegration during thermo-oxidative aging. The change trend of dielectric properties is first declining, and then rising, finally stable. In the four kinds of materials, epoxy composite has the worst electrical insulation properties and biggest change of dielectric parameters at different aging state. BMC and GPO-3 have relatively stable dielectric properties.

(2) The weak bond of the high polymer in composite materials is easily to react with oxygen. In the four kinds of materials, the epoxy composite has the highest melting temperature, and GPO-3 is the second. The crystallization of BMC is the most perfect. The area of GPO-3's melting peak is the largest, which means it has the best flame retardant performance.

(3) The main reasons of the insulation faults in high voltage switchgear are high temperature and flashover. It requires the insulation materials with lower dielectric loss, higher flame retardant performance and excellent thermal stability performance. The appearance of the glass fiber significantly improved the insulating properties and anti-aging properties of composite materials. The study in this paper can provide a strong basis to the selection of insulation material.

\section{Acknowledgments}

This work was supported by the Science and Technology Program of Jiangsu Electric Power Company (J2014035) and the National Natural Science Foundation of China (51407026).

\section{References}

[1] Wang Xiaohua, Rong Mingzhe. et al. Development of On-line Monitoring System of Medium Voltage Switchgear[J]. High Voltage Apparatus, 2009 45(3): 52-55

[2] Pan Changming, Liu Gang, et al. High Voltage Switchgear Insulation Accident's Analysis and Countermeasures[J]. High Voltage Apparatus, 2011, 47(7): 90-93.

[3] Wang Jun. Analysis on Insulation Mode and Fault of Medium Voltage Switchgear[J]. Insulating Materials, 2008, 41(3): 15-20.

[4] Peng Yun. The Research of the Online Temperature Monitor for 10kV Switch Assemblies[D]. South China University of Technology, 2010.

[5] Li Shuangna. Formulation and Properties Study of Glass Fiber/Unsatuated Polyester Resin Composite Materials[D]. Harbin Institute of Technology, 2012.

[6] Qi Shuangchun, Lan Liqin, et al. Recent Advances In Research of Flame Retardant Unsaturated Polyester Resins[J]. Thermosetting Resin, 2011, 26(2): 55-59.

[7] Robert I M. Advanced composite structures research in Australia[J]. Composite Structures, 2002, 57: 3-10.

[8] Wang Cancan, Yue Ping, et al. Electrical Properties Study of Epoxy Resin Composite Material Applied in Transmission Tower[J]. Insulating Materials, 2014, 47(2): 36-40. 